\title{
ENUNCIAÇÕES AVALIATIVAS DE ACOMPANHANTES SOBRE O CUIDADO PRESTADO À MULHER
}

\author{
EVALUATIVE STATEMENTS OF ESCORTS ABOUT THE \\ CARE PROVIDED TO WOMEN
}

\section{DECLARACIONES EVALUATIVAS DE ESCORTS ACERCA DE LA ATENCIÓN PRESTADA A LAS MUJERES}

\author{
Keury Thaisana Rodrigues dos Santos Lima ${ }^{1}$ \\ Telmara Menezes Couto ${ }^{2}$ \\ Jaqueline Alves Pires ${ }^{3}$ \\ Patrícia Santos de Oliveira ${ }^{4}$ \\ Laís Teixeira Silva Almeida ${ }^{5}$ \\ Aiara Nascimento Amaral Bomfim ${ }^{6}$ \\ Daiane Teixeira Soares ${ }^{7}$
}

Como citar este artigo: Lima KTRS, Couto TM, Pires JA, Oliveira PS, Almeida LTS, Bomfim ANA, et al. Enunciações avaliativas de acompanhantes sobre o cuidado prestado à mulher. Rev baiana enferm. 2021;35:e42393.

Objetivo: descrever as enunciações avaliativas de acompanhantes sobre o cuidado prestado às mulheres em processo de parto. Método: pesquisa descritiva com abordagem qualitativa realizada com dez acompanhantes em uma maternidade escola. Utilizou-se a entrevista semiestruturada como técnica de coleta de dados. Para o tratamento das falas, empregou-se a Análise de Conteúdo Temática Categorial. Resultados: o conteúdo obtido foi sistematizado em duas categorias: enunciações avaliativas relacionadas aos serviços de saúde e enunciações avaliativas relacionadas ao cuidado prestado às mulheres em processo de parto. Considerações Finais: as(os) acompanhantes enunciaram e vivenciaram o cuidado prestado muitas vezes como limitado e associado à sobrecarga de trabalho dos profissionais, entretanto reforçaram o acolhimento e a comunicação como importantes.

Descritores: Acompanhantes Formais em Exames Físicos. Parto. Serviços de Saúde. Pessoal de Saúde. Avaliação em Saúde.

Objective: describing the evaluative statements of escorts about the care provided to women in the process of birth. Method: a descriptive research with qualitative approach performed with ten escorts in a maternity school. The semi-structured interview was used as a data collection technique. For the treatment of the statements, the Thematic Content Analysis Categorial was used. Results: the content obtained was systematized into two categories: evaluative statements related to health services and evaluative statements related to the care provided to women in the process of birth. Final thoughts: the escorts enunciated and experienced the care provided many times as limited and associated with the workload of the professionals; however, they reinforced the reception and communication as important.

Descriptors: Formal Escorts in Physical Examinations. Parturition. Health Services. Health Personnel. Health Assessment.

\footnotetext{
Enfermeira. Mestre em Enfermagem. Universidade Federal da Bahia. Salvador, Bahia Brasil. keury_rodrigues@hotmail.com. http://orcid.org/0000-0003-0768-I I 04. Enfermeira. Doutora em Enfermagem. Professora na Universidade Federal da Bahia. Salvador, Bahia, Brasil. https://orcid.org/0000-000 I-6836-8563. 
Objetivo: describir las declaraciones evaluativas de los acompañantes acerca la atención prestada a las mujeres en el proceso de parto. Método: se trata de una investigación descriptiva con enfoque cualitativo realizada con diez acompañantes en una escuela de maternidad. La entrevista semiestructurada se utilizó como técnica de recopilación de datos. Para el tratamiento de las declaraciones, se utilizó el Categorial de Análisis Temático de Contenido. Resultados: el contenido obtenido se sistematizó en dos categorías: declaraciones evaluativas relacionadas con los servicios de salud y declaraciones evaluativas relacionadas con la atención prestada a las mujeres en el proceso de parto. Consideraciones finales: los acompañantes enunciaron y experimentaron la atención prestada muchas veces tan limitada y asociada con la sobrecarga de trabajo de los profesionales, sin embargo, reforzaron la recepción y la comunicación como importante.

Descriptores: Compañeros Formales en Exámenes Físicos. Parto. Servicios de Salud. Personal de Salud. Evaluación de la Salud.

\section{Introdução}

A presença da(o) acompanhante durante o processo de parto e nascimento sempre foi um evento vivenciado por mulheres ao longo da história, quando este ainda era uma experiência íntima que ocorria no ambiente familiar. Tal evento tinha o apoio de outras mulheres, as quais se dedicavam a proporcionar à parturiente uma vivência confortável ${ }^{(1)}$.

Com a institucionalização do parto, a intensa medicalização e intervenções cirúrgicas passaram a fazer parte do cenário de parturição. Por conseguinte, a hospitalização desse rito de passagem, que era vivenciado no âmbito doméstico, resultou em maior distanciamento dessas pessoas mais íntimas da mulher do cenário de nascimento $^{(2)}$. Ainda que confirmada cientificamente a importância do apoio social e contínuo no processo de parto, por muito tempo mulheres experienciaram a dor e a vulnerabilidade de estar sem esse suporte, e essa(e)s acompanhantes foram privados de vivenciar, sob sua ótica, esse momento de maneira singular ${ }^{(3)}$.

O apoio contínuo durante esse processo afeta as experiências da mulher e o resultado do nascimento. Desse modo, os profissionais de saúde, em especial as enfermeiras obstetras, devem buscar estratégias para reinserir a(o) acompanhante no cenário de parto e nascimento, para que possa atuar ativamente nesse processo e, com isso, possibilitar benefícios à mulher. Além disso, essas pessoas, ao se aliarem ao trabalho da Enfermeira Obstetra, podem potencializar o cuidado prestado e gerar impacto positivo no processo de trabalho dessa profissional $^{(4-5)}$.
O Ministério da Saúde reconhece os benefícios trazidos pela presença da(o) acompanhante e que as gestantes que contam com essa participação no parto e puerpério imediato ficam mais tranquilas e seguras durante o processo. Para garantir a presença dessa pessoa escolhida pela mulher, foi sancionada, no Brasil, a Lei n. 11.108/2005, conhecida como Lei do Acompanhante $^{(6)}$. Embora a presença de acompanhante seja garantida pela legislação, observam-se, em alguns serviços de saúde, obstáculos à sua participação, justificados pela inadequada infraestrutura e, principalmente, pela falta de preparo da equipe de saúde no que diz respeito à lei ${ }^{(7)}$.

Evidências apontam que a presença de acompanhante pode ter efeito protetor, dificultando os excessos de interferências e procedimentos, muitas vezes desnecessários e até prejudiciais no processo parturitivo, bem como reduzindo os atos de violências obstétricas por parte da equipe assistencial ${ }^{(4)}$. Nessa perspectiva, a presença de uma figura conhecida pela mulher estimula uma relação mais cordial por parte da equipe prestadora da assistência. Ademais, também traz benefícios de suporte no trabalho de parto e parto para os desfechos perinatais, como, por exemplo, menor duração do trabalho de parto e proporção de cesarianas, assim como menor uso de analgesia ${ }^{(4-8)}$.

Nesse cenário, observa-se que tal situação reverbera na avaliação de mulheres e acompanhantes acerca dos serviços de saúde e do cuidado prestado por profissionais nas instituições hospitalares, bem como nos impactos desse 
cuidar para o próprio profissional ${ }^{(1)}$. Estudos apontam a crescente tendência para pesquisas de avaliação em saúde, sobretudo no que diz respeito à avaliação dos efeitos de políticas específicas, como é o caso das políticas de saúde para as mulheres, principalmente sob a ótica das usuárias dos serviços. Isto pode permitir a intervenção sobre problemas reais apresentados por esse segmento populacional, bem como para a construção de estratégias de manutenção de acompanhantes dessas mulheres nos cenários de parto e nascimento ${ }^{(7)}$.

A avaliação, na perspectiva de profissionais de saúde e de usuárias do serviço, é considerada elemento significativo no desenvolvimento dos processos assistenciais e gerenciais. Entretanto, a avaliação por acompanhantes de mulheres acerca do cuidado que é prestado nas instituições é pouco relatada e priorizada dentro das políticas públicas de saúde, sobretudo em maternidade $^{(5)}$. Todavia, ainda que hoje já estejam presentes no cenário de parto institucionalizado e respaldados pela lei, esses atores sociais não estão amparados por ações que propiciem a melhoria de sua permanência nos serviços de saúde.

Nesse sentido, por meio de resultados de estudos que evidenciem essas características avaliativas, em especial aqueles que demonstrem a ótica de acompanhantes, é possível reorganizar os serviços de saúde, a fim de atender as demandas desses usuários que integram as estratégias e as políticas de atenção à saúde da mulher no Sistema Único de Saúde, com vistas a melhorar o cuidado prestado. Assim, diante do que se recomenda para o cotidiano das instituições que atendem à parturiente e o que é vivenciado por ela e seu apoio social, o objetivo do presente estudo é descrever as enunciações avaliativas de acompanhantes sobre o cuidado prestado às mulheres em processo de parto.

\section{Método}

Estudo descritivo com abordagem qualitativa. Realizado em uma maternidade escola de médio porte localizada na cidade de Salvador, Bahia, Brasil, com acompanhantes de mulheres em processo de parto.
A seleção dos participantes foi feita por meio da lista de acompanhantes presentes na instituição no momento das visitas e que aceitaram participar da pesquisa. Foi adotado como critério de inclusão acompanhantes de mulheres que foram internadas em processo de parto, podendo ser familiar ou não e que tivesse acompanhado durante todo esse processo. Como critério de exclusão estabeleceu-se: acompanhante que não teve condições físicas e/ou emocionais declaradas à pesquisadora de participar da pesquisa. Os aceites totalizaram 11, entretanto a amostra deste estudo foi composta por 10 acompanhantes, devido ao critério de exclusão.

A maternidade onde se deu a coleta de dados é uma unidade docente-assistencial de Obstetrícia, Neonatologia e Saúde Perinatal. É considerada hospital de médio porte, tipo específico de média complexidade, com 80 leitos. Realiza uma média de 250 partos e mais de 6 mil procedimentos ambulatoriais por mês. A unidade presta serviços de urgência e emergência obstétrica e neonatal 24h, oferecendo Acolhimento com Classificação de Risco de pacientes provenientes do Ambulatório da instituição e da Central Estadual de Regulação do estado da Bahia. Como referência para o pré-natal de alto risco, também disponibiliza vagas para a rede voltadas para mulheres residentes em cidades da Região Metropolitana de Salvador (RMS) e do interior da Bahia.

A Maternidade conta com equipe multiprofissional e oferece atendimentos ambulatoriais e hospitalares em Cardiologia, Clínica Médica, Educação Física, Endocrinologia, Endoscopia Ginecológica, Fisioterapia Geral, Fisioterapia em Saúde da Mulher, Fonoaudiologia, Genética Médica, Ginecologia, Hematologia, Mastologia, Medicina Fetal, Neonatologia, Neuropediatria, Nutrição, Obstetrícia, Oftalmologia, Psicologia, Psiquiatria, Serviço Social e Terapia Ocupacional, dentre outros serviços de apoio à assistência à saúde da mulher e da criança. Conta ainda com Unidade de Terapia Intensiva Neonatal (UTIN), Unidade de Cuidados Intermediários Neonatais Convencional (UCINCo) e Unidade de Cuidados Intermediários Neonatais Canguru (UCINCa), Banco de Leite Humano (BLH) e Casa da Gestante, Bebê e Puérpera (CGBP). 
A coleta de dados ocorreu entre os meses de agosto e novembro de 2018 durante visitas da pesquisadora que conduziu as entrevistas na instituição. As entrevistas foram encerradas, tomando-se por base o critério de saturação dos dados, na décima entrevista. A delimitação do número de entrevistados ocorreu quando o conteúdo foi suficiente para permitir o aprofundamento, a abrangência e a diversidade do processo de compreensão das enunciações descritas. A entrevista semiestruturada contou com questões objetivas referentes à caracterização dos participantes, além da seguinte questão norteadora: Como foi para você vivenciar o processo de parto?

As entrevistas foram realizadas em salas privativas na própria maternidade, em horários previamente agendados de acordo com a disponibilidade dos participantes, sendo gravadas com o auxílio de um smartphone e teve duração média de 40 minutos. Procedida esta etapa, as entrevistas foram transcritas na íntegra e receberam códigos de identificação abreviados, em que a letra "E" refere-se a "Entrevista" seguida do numeral arábico correspondentes à ordem da realização (E1, E2, E3 etc.), com vistas a preservar o anonimato dos participantes.

Os dados foram organizados com base na Análise do Conteúdo Temático-Categorial proposta por Franco ${ }^{(9)}$. Dessa forma, as falas foram submetidas ao processo de unitarização, o qual requereu uma releitura das mensagens atribuídas ao objeto de estudo, favorecendo a identificação e a definição das unidades de análise (conceitos frases, temas ou palavras). Estas foram agregadas em unidades de registro e, posteriormente, nas unidades de contexto.

As falas dos participantes foram analisadas sob os pressupostos teóricos das normativas ministeriais e leis atuais, o que permitiu a identificação das categorias de análise. Das falas da(o)s acompanhantes emergiram suas vivências e as enunciações avaliativas acerca do cuidado prestado às mulheres em processo de parto. Os achados foram relevantes e originais para a pesquisa e para as análises que permitiram.

O estudo teve anuência da instituição e foi desenvolvido após apreciação e aprovação do projeto de pesquisa pelo Comitê de Ética e Pesquisa (CEP) da Maternidade Climério de Oliveira sob Parecer n. 2320722. Além disso, atendeu às diretrizes e normas da Resolução n. 466/2012 do Conselho Nacional de Saúde, que regulamenta a pesquisa envolvendo seres humanos. Todos os participantes foram esclarecidos sobre o estudo e assinaram o Termo de Consentimento Livre e Esclarecido (TCLE). Também foram informados sobre as medidas para assegurar o anonimato e a confidencialidade dos dados obtidos.

\section{Resultados}

Participaram do estudo dez acompanhantes de mulheres em processo de parto, sendo sete homens e três mulheres. Destes, seis encontravam-se na faixa etária de 18 a 34 anos e quatro na faixa etária de 35 a 59 anos. Acerca do grau de parentesco, os homens declararam relação marital e entre as três mulheres, duas eram a mãe e uma, a irmã. Todos os participantes declararam-se da raça negra (pretos e/ou pardos).

As falas da(o)s acompanhantes foram agrupadas em duas categorias: Enunciações avaliativas relacionadas aos serviços de saúde e Enunciações avaliativas relacionadas ao cuidado prestado às mulheres em processo de parto.

\section{Enunciações avaliativas relacionadas aos serviços de saúde}

Nas enunciações avaliativas da(o)s acompanhantes de mulheres em processo de parto foi referida a falta de capacidade dos serviços de saúde no âmbito público comparado aos serviços ofertados na rede privada. $\mathrm{A}(\mathrm{O}) \mathrm{s}$ acompanhantes culparam o governo pela falta de investimentos:

\footnotetext{
Alguns hospitais não têm capacidade de atender muitas pessoas, isso é complicado. Minha esposa sentiu muito medo, por não termos pensado antes em ter feito um plano de saúde e agora nos deparamos em ter que ir para o SUS. Acredito que a culpa é do governo, por não fazer investimentos. (E1).
}

As falas sugerem que a capacidade de atendimento nos serviços de saúde está associada ao processo de superlotação nas unidades. 
Essa sobrecarga nos serviços tem afastado a(o)s acompanhantes do processo de parto e gerado medo. Em paralelo, eles se sentiam seguros quando havia uma estrutura adequada e responsabilização do serviço pela paciente. Todavia, referiram o atendimento, pela estrutura institucional, das necessidades básicas da(o)s acompanhantes em consonância com a segurança da paciente, bem como a importância da oferta de informação sobre a localização dos setores:

Na maternidade não tinha vaga. Então, os profissionais se responsabilizaram de regular minha irmã. Conseguiram a vaga em outro hospital, então ocorreu tudo bem. (E10).

No outro bospital que estávamos foi um momento constrangedor, uma carnificina. O acompanhante não podia entrar e saber como está a mulber por conta de superlotação. É difícil acompanhar o parto da mulher. Eu fiquei com bastante medo, mas quando encaminharam minha filha para esse bospital, eu senti segurança. Aqui tem uma estrutura maravilhosa! O banheiro, os aparelhos todos bons, mas deveria ter indicação de onde ficam os lugares, o refeitório, o banheiro. (E9).

O banho do acompanhante tem que ser discutido. Estávamos na rua e agora ficamos na maternidade desde que chegamos sem tomar banbo. Queremos nos refrescar e ainda tem a questão das bactérias. (E8).

As enunciações avaliativas da(o)s acompanhantes atribuíam o melhor atendimento ofertado no serviço de saúde da capital quando comparado à assistência prestada no interior do estado. Referiram o momento do transporte como constrangedor e desconfortável:

Foi um parto muito complicado, foi dificil para o bebê sair. Ela só não morreu, porque foi transferida do interior para a capital. Eu agradeço muito pelo atendimento. (E3).

Os profissionais fizeram todo o procedimento correto. Não teve nenbuma intercorrência. Presenciei o atendimento com a paciente, a criança e gostei. (E10).

Agradeci a Deus, por permitir que minha filha chegasse na maternidade da capital. Na viagem, a ambulância sacudia e foi muito constrangedor. Ela com aquele barrigão, sentindo dor, sem conforto algum. (E2).

\section{Enunciações avaliativas relacionadas ao cuidado prestado às mulheres em processo de parto}

As falas enunciaram pessoas sem condições e desprezadas no processo de cuidado. A(O)s acompanhantes compartilharam momentos de tensão sobre a vivência do profissional com a sobrecarga de trabalho, ao atender mulheres que chegavam estressadas nos serviços de saúde e transferiam para o profissional toda a carga emocional do momento:

É a primeira vez que presenciei pessoas sem condiçoes, sendo desprezadas. É uma tensão muito grande para o profissional de saúde que atende muita gente e com vários problemas. As pessoas chegam estressadas, descontam nos profissionais, e esses, sobrecarregados, acabam não sendo respeitados no serviço de saúde. Eu não acho isso certo. Tem que pensar nos dois lados: o profissional e a paciente. (E1).

O depoimento a seguir mostra apreço da(o)s acompanhantes pelo acolhimento prestado por profissionais, entretanto relataram desconforto, devido ao número elevado de estudantes e questionamentos no momento do parto. $\mathrm{A}(\mathrm{O}) \mathrm{s}$ acompanhantes atribuíram a presença de muitos estudantes ao fato de ser uma rotina comum em um hospital:

\footnotetext{
Na maternidade, ela foi bem recebida pela equipe médica e de enfermagem. Todos foram ótimos! Não tenbo o que reclamar sobre isso, mas achei muito desconfortável a pessoa estar sentindo dor com a sala cheia de estagiários. Uma estudante fez muitas perguntas para minha filha; ela não queria falar nada. Ela estava sentindo muita dor e queria pedir para a estudante ir embora, mas é um hospital. (E2).
}

As enunciações revelaram a importância de profissionais escutarem a mulher e estarem presentes no processo de parto. A ausência desses pode gerar falta de cuidado e medo, na visão da(o)s acompanhantes:

\section{Na hora que eu não estava, minha esposa relatou que as enfermeiras diziam que ela estava fazendo escândalo. Eu queria dizer para elas ouvirem a paciente, porque isso é importante. (E5).}

Eu estava sozinho com minha esposa e logo veio em meu pensamento que as médicas e as enfermeiras não queriam ajudar naquele momento. Eu tive medo dela desmaiar, porque elas estavam na recepção. Apesar disso, elas estavam sempre de olho, entrando e saindo da sala para ver como ela estava, (E4).

$\mathrm{A}(\mathrm{O}) \mathrm{s}$ acompanhantes referiram que a assistência para a gestante de risco poderia ser melhor desde o diagnóstico da complicação até a transferência para o hospital. No momento do parto, a(o)s acompanhantes sentiam-se inúteis diante da situação de complicação na presença da equipe: 
A assistência poderia ter sido melbor, já que era uma gestante de risco. Eu não entendo muito bem. Foi diagnosticado no ambulatório que o coração do bebê estava baixo. Acho que deveriam ter providenciado um carro de imediato, para transferir ela para o bospital. Mas, na hora do parto, a equipe estava muito presente $e$, nesse momento, me senti uma pessoa inútil. (E7).

$\mathrm{A}(\mathrm{O}) \mathrm{s}$ acompanhantes enalteceram a comunicação dos profissionais de saúde e relataram sua participação no processo de parto como uma oportunidade de conhecimento referente ao atendimento e aos procedimentos prestados pelos profissionais:

Eu gostei do atendimento dessa maternidade e dos profissionais. Todos falam com a gente. Os médicos e os enfermeiros passam, cumprimentam com bom dia, mesmo para quem não conbece. (E3).

É bom quando o profissional chega para o acompanhante e explica quando o parto está demorando, ou porque não fez uma cesariana. É importante ouvir dos profissionais que estão buscando o melhor para a paciente e a criança. Eu observei isso a todo momento. (E10).

É a primeira vez que participo de um processo de parto. Foi importante para mim ver como é o atendimento, como a criança nasce, como os profissionais trabalham e saber dos procedimentos. (E6).

\section{Discussão}

No que se refere à aplicabilidade prática, percebe-se que a inserção da(o) acompanhante no cenário de parto, conforme prescrito na lei, permitiu um olhar diferenciado deste que é usuário dos serviços e vivencia o processo de parto sob outra perspectiva, não somente a de espectador, mas também de avaliador.

As enunciações avaliativas da(o)s acompanhantes de mulheres em processo de parto revelaram como a falta de investimentos repercute no setor saúde, principalmente nas instituições públicas e nos processos de trabalho. Isso contribui para a precarização do serviço de saúde pública, a qual se fortalece com iniciativas governamentais de redução dos recursos destinados à saúde $^{(10)}$. Tendo em vista situações como essa, a aprovação da Proposta de Emenda Constitucional, em 2016, permitiu congelar investimentos do Estado na saúde por 20 anos, o que está em desacordo com as necessidades da população e as diretrizes do Sistema Único de Saúde (SUS) ${ }^{(11)}$.

$\mathrm{A}(\mathrm{O}) \mathrm{s}$ acompanhantes, em suas enunciações avaliativas, ao comparar os serviços prestados no âmbito público e privado, refletiram sobre a incapacidade da oferta nos serviços públicos. Este achado apoia-se na realidade do subfinanciamento e da má distribuição de recursos que aceleram a precarização no SUS. Essa situação tem alimentado, no imaginário da sociedade, o entendimento de que há maior competência nos investimentos da iniciativa privada no setor saúde. Isso, entretanto, pode viabilizar politicamente o surgimento de propostas de coparticipação ou inserção do capital privado no SUS e contribuir para as privatizações e a mercantilização da saúde ${ }^{(12-13)}$.

As comparações entre os serviços de saúde público e privado têm gerado insegurança na(o)s acompanhantes, ao utilizarem os serviços disponibilizados pelo SUS. Essa situação pode ocorrer devido à tendência da população de valorizar os serviços de saúde prestados pela rede privada como mais qualificados, com base no acesso aos planos de saúde ${ }^{(13)}$.

Essa compreensão foi verbalizada nos depoimentos da(o)s acompanhantes em situações que geraram temor e constrangimento frente à superlotação nos serviços públicos. Resultado de estudo sobre gastos públicos com saúde mostrou que a(o)s acompanhantes culpabilizaram o governo pela superlotação das maternidades públicas, ausência de vagas e, consequentemente, pela sobrecarga de trabalho para os profissionais de saúde ${ }^{(10)}$.

Os problemas oriundos da superlotação em maternidades públicas, no Brasil, são crescentes. Isso tem acarretado frequente publicização de denúncias a respeito das dificuldades enfrentadas pelas parturientes nas maternidades públicas, principalmente no que se refere à insuficiência de leitos, de profissionais e de instalações físicas precárias para atenção ao parto e nascimento ${ }^{(14)}$.

Essa insuficiência na oferta de leitos, de materiais e de recursos humanos torna-se notória, por exemplo, nas solicitações de transferência das parturientes de unidades de saúde do interior para a capital. Devido à organização na oferta e distribuição dos leitos obstétricos, há maior disponibilidade desses serviços nos grandes centros urbanos, onde se concentram também 
maior aporte tecnológico e recursos humanos mais capacitados ${ }^{(15)}$.

Dentre os serviços de saúde que estruturam o atendimento à mulher, destaca-se o transporte. $\mathrm{A}(\mathrm{O}) \mathrm{s}$ acompanhantes referiram a insegurança nas ambulâncias, durante o transporte da mulher em trabalho de parto do interior para a capital, e enalteceram a possibilidade de transferência da mulher para maternidades que possuíam qualidade na estrutura, nos recursos materiais e permitiam a presença de acompanhante. Esta realidade vem contribuindo para a superlotação nas unidades hospitalares dos grandes centros e confirmam a tendência da atual política de ajuste estrutural do Estado, que tem refletido nos investimentos para a saúde ${ }^{(10,15)}$.

$\mathrm{A}(\mathrm{O}) \mathrm{s}$ acompanhantes revelaram, em suas enunciações, a importância da estrutura física adequada nas maternidades, principalmente relacionada à oferta de insumos e banheiros, o que implica na satisfação dos usuários. Estudo realizado na cidade de Recife (PE) sobre a qualidade do atendimento ao parto na rede pública hospitalar revelou que a insatisfação de usuários esteve mais relacionada com a ambiência das maternidades ${ }^{(15)}$. Entretanto, a estruturação da rede obstétrica configura-se em uma problemática para as maternidades, já que, além de ocasionarem superlotação do serviço, aumentam as demandas assistenciais e, consequentemente, a sobrecarga de trabalho para os profissionais, o que tem afetado a saúde desses indivíduos ${ }^{(16-17)}$.

Pesquisas brasileiras abordam a sobrecarga de trabalho como consequência do aumento das demandas assistenciais e administrativas, que dificultam o planejamento e a execução das ações da assistência a ser prestada. Esse excesso na carga normal de trabalho, tanto física quanto psíquica, somada às formas de organização do trabalho, pode refletir diretamente na vida dos profissionais e em prejuízos na assistência prestada às mulheres, contribuindo para a insatisfação ${ }^{(14,18)}$. Essa carga desmedida pode ser mais intensa para o pessoal de enfermagem, por participar no cuidado direto ao paciente e atuar na linha de frente desse cuidado ${ }^{(19)}$.

A sobrecarga de trabalho dos profissionais de saúde pode favorecer a permanência de um número elevado de estudantes no cenário do parto, uma vez que, estando sobrecarregados em suas funções assistenciais, administrativas e, inclusive, de preceptoria, não conseguem acolher e orientar adequadamente os estudantes em formação dentro do serviço. Esse fato pode impactar na formação dos discentes, deixando-os expostos ao desenvolvimento de práticas assistenciais desfavoráveis ${ }^{(19-20)}$. Neste sentido, a(o)s acompanhantes relataram desconforto com o grande número de estudantes presentes no cenário de parto, inquirindo as parturientes em momentos inoportunos e causando constrangimento para as mulheres, por se sentirem invadidas em sua privacidade.

Essa situação permite ponderar sobre a formação acadêmica alicerçada na valorização de padrões tecnicistas na obstetrícia. No entanto, o que se verifica é um processo de ensino-aprendizagem que carece estar baseado nas particularidades e necessidades da mulher e de sua(seu) acompanhante. Desse modo, os profissionais de saúde precisariam aprender, em sua formação, que o parto é um evento natural e deve ter a sua fisiologia respeitada por meio de práticas benéficas, humanizadas e cientificamente comprovadas.

Quanto aos sentimentos no momento no parto, a(o)s acompanhantes relataram sentir-se inúteis em situação de intercorrência obstétrica. Essa sensação pode estar atrelada à percepção de não pertencimento ao cenário juntamente com a mulher e/ou não se sentirem integrados pela equipe de saúde ${ }^{(19)}$. Esse afastamento, além de provocar sentimentos desagradáveis, configura-se como uma violação do direito garantido por lei ${ }^{(8)}$. Apesar disso, a(o) acompanhante entende a relevância da sua presença no momento do parto, bem como da necessidade de conhecimento quanto aos procedimentos e ao atendimento em saúde.

Cabe salientar que os participantes da pesquisa valorizaram, em seus enunciados, a comunicação a eles dirigida pelos profissionais, a qual pode favorecer o atendimento de suas demandas específicas na condição de usuários dos serviços de saúde. Neste sentido, é importante destacar as ações propostas pela Política 
Nacional de Humanização, dentre as quais destaca-se a valorização da escuta, do vínculo e das relações afetivas na prática de profissionais de saúde. Tais ações facilitam o processo de comunicação com usuários dos serviços, de modo que as informações possam ser apreendidas sem falhas, dúvidas ou mesmo não entendimento ${ }^{(14)}$. Por essa razão, a comunicação representa uma importante ferramenta para melhorar a assistência em saúde. Vale ressaltar que a qualidade da comunicação entre profissionais de saúde e usuários dependerá da disponibilidade do profissional para estabelecer essa comunicação com o outro ${ }^{(20)}$.

A comunicação, portanto, favorece a proximidade entre as pessoas e, particularmente no ambiente do parto, estabelece-se entre profissional e mulher. Os participantes desta pesquisa revelaram medo e consideraram falta de cuidado quando profissionais não estavam acompanhando a mulher durante todo o processo de parto. Estudo realizado no estado do Paraná, Brasil, em 2013, com 11 mulheres e 11 acompanhantes, mostrou a importância de ter profissionais de saúde disponíveis e auxiliando o cuidado quando solicitado. Ademais, aspectos como atenção dispensada, humor, vínculo criado, disponibilidade de ajuda, apoio ao aleitamento materno e cuidados com o bebê foram avaliados de forma positiva diante da assistência prestada às mulheres em processo de parto ${ }^{(8)}$.

Esta pesquisa possibilita uma ampliação de olhares das políticas em saúde da mulher e sua aplicabilidade na prática, bem como contribui para mais visibilidade para este ator tão importante no cenário do parto. Todavia, são poucas as situações de escuta e fala da(o) acompanhante. O estudo apresentou limitações no que tange ao conteúdo das falas, número de participantes, já que estes trazem consigo características familiares, sociais, culturais e de gênero particulares. Além disso, a pesquisa foi composta por acompanhantes de uma maternidade escola, fato que permite sugerir avaliações da realidade dessa instituição em relação ao cuidado recebido no serviço e por profissionais de saúde.

\section{Considerações finais}

Os achados deste estudo permitem afirmar que acompanhantes de mulheres em processo de parto referenciam o cuidado prestado muitas vezes limitado em decorrência da sobrecarga de trabalho dos profissionais. Esses atores sociais enunciam a tensão de vivenciar esse momento junto às mulheres, entretanto reforçam o apreço pelo acolhimento dispensado no momento do encontro. Alguns sentimentos, como desconforto, aliados ao excesso de profissionais e estudantes no cenário de parto e nascimento, bem como os sentimentos de inutilidade frente às complicações obstétricas, despontaram nas falas dos participantes. A pesquisa demonstrou ainda, por meio das enunciações da(o)s acompanhantes, a necessidade de melhor estruturação física dos espaços com vistas à promoção de um acolhimento também a elas(es). É importante destacar que um dos resultados apontados foi a satisfação quando os profissionais comunicavam-se com a mulher e a(o) acompanhante e estavam presentes no parto, assistindo a mulher em todo o processo. Contudo, mais pesquisas sobre acompanhantes de mulheres em processo de parto são necessárias, para possibilitar reformulações de ações a serem desenvolvidas com base em evidências e ratificar a importância de condutas profissionais mais humanizadas no cenário do parto.

\section{Colaborações:}

1 - concepção, projeto, análise e interpretação dos dados: Keury Thaisana Rodrigues dos Santos Lima, Telmara Menezes Couto e Patrícia Santos de Oliveira;

2 - redação do artigo e revisão crítica relevante do conteúdo intelectual: Keury Thaisana Rodrigues dos Santos Lima, Jaqueline Alves Pires, Patrícia Santos de Oliveira, Laís Teixeira Silva Almeida, Aiara Nascimento Amaral Bomfim e Daiane Teixeira Soares;

3 - aprovação final da versão a ser publicada: Keury Thaisana Rodrigues dos Santos Lima. 


\section{Referências}

1. Bohren MA, Hofmeyr G, Sakala C, Fukuzawa RK, Cuthbert A. Continuous support for women during childbirth. Cochrane Database Sys Rev. 2017;7:(CD003766). DOI: http://dx.doi. org/10.1002/14651858.CD003766.pub6

2. Andrade LO, Felix ESP, Souza FS, Gomes LOS, Boery RNSO. Practices of nursing professionals against humanized labor 2017;11(6):2576-85. DOI: https://doi.org/10.5205/reuol.9799-86079-1RV.1106sup201712

3. Sousa AMM, Souza KV, Rezende EM, Martins EF, Campos D, Lansky S. Practices in childbirth care in maternity with inclusion of obstetric nurses in Belo Horizonte, Minas Gerais. Esc Anna Nery. 2016;20(2):324-31. DOI: https:// doi.org/10.5935/1414-8145.20160044

4. Pintassilgo S, Carvalho H. Trends and consequences of the technocratic paradigm of childbirth in Portugal: a populationbased analysis of birth conditions and social characteristics of parents. Sex Reprod Healthc. 2017;13:58-67. DOI: https://doi.org/10.1016/j. srhc. 2017.06 .003

5. Carvalho CFS, Carvalho IS, Brito RS, Vitor AF, Lira ALBC. The partner as a caregiver in the birth process. Rev Rene. 2015;16(4):613-21. DOI: https://doi.org/10.15253/2175-6783.2015 000400019

6. Gomes IEM, Mello PSM, Langendorf TF, Paula CC, Gomes CA, Ribeiro AC. Benefits of the presence of a companion during the process of labor and delivery: integrative review. Rev Enferm UFSM. 2019;9:1-18. DOI: $10.5902 / 2179769234170$

7. Batista $\mathrm{BD}$, Bruggemann $\mathrm{OM}$, Junges $\mathrm{CF}$, Velho MB, Costa R. Factors associated with the birth companion's satisfaction with the care provided to the parturient woman. Cogitare Enferm. 2017;22(3):e51355. DOI: http://dx.doi. org/10.5380/ce.v22i3.51355

8. Almeida AF, Brüggemann $\mathrm{OM}$, Costa R, Junges CF. Separation of the woman and her companion during cesarean section: a violation of their rights. Cogitare Enferm. 2017;(23)2:e53108. DOI: http://dx.doi.org/10.5380/ce.v23i1.53108

9. Franco MLPB. Análise de conteúdo. Brasília: Liber Libro; 2018.
10. Saldiva PHN, Veras M. Gastos públicos com saúde: breve histórico, situação atual e perspectivas futuras. Estud Av. 2018;32(92):47-61. DOI: https://doi.org/10.5935/0103-4014.20180005

11. Rossi P, Dweck E. Impacts of the New Fiscal Regime on health and education. Cad Saúde Pública. 2016;32(12):e00194316. DOI: https://doi. org/10.1590/0102-311x00194316

12. Oliveira VJ, Penna CMM. Ethos and pathos in the delivery room. Rev Gaúcha Enferm. 2017;38(2):e67761. DOI: https://doi. org/10.1590/1983-1447.2017.02.6776

13. Leal MC, Pereira APE, Nakamura-Pereira M, Torres JA, Domingues RMSM, Dias MAB, et al. Provider-Initiated Late Preterm Births in Brazil: Differences between Public and Private Health Services. PloS One. 2016;11(5):e0155511. DOI:10.1371/journal.pone.0155511

14. Dodou HD, Sousa AAS, Barbosa EMG, Rodrigues DP. Sala de parto: condições de trabalho e humanização da assistência. Cad Saúde Colet. 2017;25(3):332-8. DOI: https:// doi.org/10.1590/1414-462x201700030082

15. Silva ALA, Mendes ACG, Miranda GMD, Souza WV. A qualidade do atendimento ao parto na rede pública hospitalar em uma capital brasileira: a satisfação das gestantes. Cad Saúde Pública. 2017;33(12):e00175116. DOI: https://doi. org/10.1590/0102-311x00175116

16. Rohr LK, Valongueiro S, Araújo TVB. Delivery care and the inadequacy of the obstetric care network in Pernambuco. Rev Bras Saúde Matern Infant. 2016;16(4):457-65. DOI: https://doi. org/10.1590/1806-93042016000400006

17. Biondi HS, Pinho EC, Kirchhof ALC, Rocha LP, Barlem ELD, Kerber NPC. Psychic workload in the process of work of maternity and obstetric centers nurses. Rev Gaúcha Enferm. 2018;(39):e64573. DOI: https://doi.org/10.1590/ 1983-1447.2018.64573

18. Soratto J, Pires DEP, Trindade LL, Oliveira JSA, Forte ECN, Melo TP. Job dissatisfaction among health professionals working in the family health strategy. Texto contexto-enferm. 2017;26(3):e2500016. DOI: https://doi.org/10. 1590/0104-07072017002500016

19. Progianti JM, Moreira NJMP, Prata JA, Vieira MLC, Almeida TA, Vargens OMC. Job insecurity among obstetric nurses. Rev Enferm UERJ. 
2018;26:e33846. DOI: http://dx.doi.org/10.12957/ reuerj.2018.33846

20. Finkler RU, Silva AS, Bonamigo AW. Vision of the preceptors on preparedness and hosting of the graduation student in primary health care.
RSD. 2019;8(2):e1182557. DOI: http://dx.doi. org/10.33448/rsd-v8i2.557

Recebido: 28 de setembro de 2020

Aprovado: 25 de março de 2021

Publicado: 26 de abril de 2021

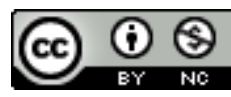

A Revista Baiana de Enfermagem utiliza a Licença Creative Commons - Atribuição-NãoComercial 4.0 Internacional.

https://creativecommons.org/licenses/by-nc/4.0/

Este artigo é de acesso aberto distribuído sob os termos da Licença Creative Commons (CC BY-NC).

Esta licença permite que outros remixem, adaptem e criem a partir do seu trabalho para fins não comerciais. Embora os novos trabalhos tenham de lhe atribuir o devido crédito e não possam ser usados para fins comerciais, os usuários não têm de licenciar esses trabalhos derivados sob os mesmos termos. 